\title{
Ginkgo biloba Extract 50 (GBE50) Ameliorates Insulin Resistance, Hepatic Steatosis and Liver Injury in High Fat Diet-Fed Mice
}

\author{
Liu $\mathrm{Li}^{1, *}$ \\ Li Yang ${ }^{\prime, *}$ \\ Feng Yang ${ }^{2, *}$ \\ Xin-lan Zhao' \\ Shengjiang $X_{u e^{3}}$ \\ Fang-hua Gong (iD ${ }^{4}$ \\ 'No.2 Department of Endocrinology, \\ Hunan Provincial People's Hospital, The \\ First Affiliated Hospital of Hunan Normal \\ University, Changsha, 410002, People's \\ Republic of China; ${ }^{2}$ No.2 Department of \\ Pharmacy, Hunan Provincial People's \\ Hospital, the First Affiliated Hospital of \\ Hunan Normal University, Changsha, \\ 410002, People's Republic of China; \\ ${ }^{3}$ Ladder Molecular Biomedical Research \\ Center, Guangzhou, 510800, People's \\ Republic of China; ${ }^{4}$ Department of \\ Nursing, Hunan Provincial People's \\ Hospital, the First Affiliated Hospital of \\ Hunan Normal University, Changsha, \\ 410002, People's Republic of China
}

*These authors contributed equally to this work

Correspondence: Shengjiang Xue Ladder Molecular Biomedical Research Center, No. 39 Zhanqian Road, Xinhua Street, Huadu District, Guangzhou, 510800, People's Republic of China Email wsjiangzi@|26.com

Fang-hua Gong

Department of Nursing, Hunan Provincial People's Hospital, the First Affiliated Hospital of Hunan Normal University, No. 61 Jiefangxi Road, Furong District, Changsha, 410002, Hunan Province, People's Republic of China

Tel +86 073 I-8476269|

Fax +86 073I-8329065

Email gfhnurse@aliyun.com
Background: Ginkgo biloba extract 50 (GBE50) has a variety of pharmacological functions such as anti-inflammatory, antioxidant and maintenance of glucose and lipid metabolism homeostasis. However, the therapeutic effects and mechanisms of GBE50 on non-alcoholic fatty liver disease (NAFLD) remain unknown. Therefore, in this study, we evaluated the therapeutic effects of GBE50 in NAFLD by using a high-fat diet (HFD) mice model.

Methods: C57BL/6J mice were fed a HFD diet for 15 weeks and were given respectively 25,50 , and $100 \mathrm{mg} / \mathrm{kg} \mathrm{GBE} 50$ daily by gavage from 3 to 15 weeks. After the administration, blood samples and liver tissues were collected for biochemical detection, histological measurement, immunohistochemistry and Western blot, respectively.

Results: We found that GBE50 treatment could ameliorate insulin resistance (IR), glucose intolerance, lipid accumulation, hepatic steatosis and liver injury in HFD-fed mice. Further mechanism exploration discovered that the hepatoprotective effects of GBE50 on NAFLD may be related to the strengthening of IRS-1 signal activation and the weakening of NF- $\mathrm{B}$, Akt and endoplasmic reticulum stress signals activation.

Conclusion: GBE50 is a potentially powerful therapeutic agent for the treatment of NAFLD.

Keywords: Ginkgo biloba extract 50, non-alcoholic fatty liver disease, insulin resistance, hepatic steatosis, inflammatory response, liver injury

\section{Introduction}

Non-alcoholic fatty liver disease (NAFLD) refers to a chronic liver disease that is caused by metabolic disorders and characterized by diffuse bullae steatosis and lipid accumulation in liver cells, excluding alcohol consumption, hepatitis virus, drugs and other specific factors that damage the liver. ${ }^{1,2}$ NAFLD is often closely related to metabolic diseases such as obesity, insulin resistance (IR), hypertension and dyslipidemia, so NAFLD is a chronic hepatic disease with metabolic abnormalities. ${ }^{3}$ Even, NAFLD is a potential factor for primary hepatocellular carcinoma. ${ }^{4}$ Moreover, due to the improvement of people's living standard and changes in lifestyle and diet structure in recent years, diseases caused by abnormal lipid metabolism are gradually increasing, and the prevalence of metabolic syndrome-related diseases, such as obesity and diabetes, was increasing day by day, resulting in a rapid rise in the prevalence of NAFLD. ${ }^{5}$ In particular, the prevalence of NAFLD in people with abdominal obesity is as high as $75-100 \% .{ }^{6}$ Globally, NAFLD affects about $25 \%$ of the adult population. ${ }^{7}$ In addition, the prevalence of NAFLD also showed an increasing trend among young 
children. ${ }^{8}$ Therefore, NAFLD has become a worldwide public health problem. Currently, the drugs used in the clinical treatment of NAFLD, such as membrane insulin sensitizers, antioxidants, biological agents, weight-loss drugs, antiinflammatory and liver-protecting drugs, are beneficial to the relief of symptoms, but cannot completely reverse NAFLD. ${ }^{9}$ Therefore, the current prevention and treatment methods for NAFLD are not very satisfactory.

The pathogenesis of NAFLD is very complex, which is mainly believed to be the disorder of hepatic lipid metabolism caused by IR and further triggered by a series of cytotoxic events such as hepatocyte inflammation, oxidative stress and liver injury. ${ }^{10}$ For example, the accumulation of long-chain fatty acids in hepatocytes and their oxidation in the endoplasmic reticulum (ER) lead to an increase in reactive oxygen species (ROS) production, which leads to oxidative stress and endoplasmic reticulum stress (ERS), and ultimately to lipid inflammation, cell damage, and apoptosis in hepatocytes. ${ }^{10}$ Therefore, hepatic lipid metabolism disorder is a key pathological change of NAFLD, and IR and ERS are the major cytotoxic events leading to NAFLD. ${ }^{10,11}$ Based on the major pathological changes in lipid metabolism, inhibition of IR and ERS have become the key targets for NAFLD treatment.

Ginkgo biloba extract 50 (GBE50) is the new Ginkgo biloba extracts, containing 44\% Ginkgo flavonoid glycosides (including quercetin and isorhamin derivatives) and 6\% lactones (including Ginkgolide A, B, C and $\mathrm{J}$, and ginkgolide). GBE50 has a wide variety of pharmacological effects including anti-oxidant, anti-inflammatory, vasodilating, maintaining lipid metabolism homeostasis, enhancing insulin sensitivity, etc. ${ }^{12,13}$ Based on the pathological characteristics of NAFLD and the pharmacological effects of GBE50, we hypothesized that GBE50 may have an antiNAFLD effect, while the effects and mechanisms of GBE50 on NAFLD remain unknown. Therefore, the present study was mainly explored whether GBE50 had a hepatoprotective effect on NAFLD and its mechanism.

\section{Materials and Methods}

\section{Experimental Animals and Experimental Design}

Six-week-old male C57BL/6J mice with SPF grade were purchased from Charles River Laboratory Animal Technology Co., Ltd. (Zhejiang, China), and kept in a humidity and temperature-controlled SPF grade animal room $(12 \mathrm{~h} \mathrm{light/dark} \mathrm{cycle,} \mathrm{the} \mathrm{room} \mathrm{temperature} \mathrm{was}$ maintained at $23^{\circ} \mathrm{C}-25^{\circ} \mathrm{C}$, humidity was maintained at $55 \% \sim 65 \%$ ). The mice were allowed free access to food and water before being given a special diet. After 5 days of adaptive feeding, mice were divided into normal control group (NC), $\mathrm{NC}+100 \mathrm{mg} / \mathrm{kg}$ GBE50 group, NAFLD model group, high fat diet (HFD) $+25 \mathrm{mg} / \mathrm{kg}$ GBE50 group, HFD+50 mg/kg GBE50 group, and HFD+100 mg/ $\mathrm{kg}$ GBE50 group, with 11 mice in each group. GBE50 was purchased from SPH Xingling Sci. and Tech. Pharmaceutical Co., Ltd (Shanghai, China). NAFLD mouse model was established by HFD induction. Mice in the $\mathrm{NC}$ group and the $\mathrm{NC}+100 \mathrm{mg} / \mathrm{kg}$ GBE50 group were fed a normal chow diet for 15 weeks. Mice in the NAFLD model group, the HFD $+25 \mathrm{mg} / \mathrm{kg}$ GBE50 group, the HFD $+50 \mathrm{mg} / \mathrm{kg}$ GBE50 group, and the HFD $+100 \mathrm{mg} / \mathrm{kg}$ GBE50 group were fed a HFD diet $(60 \%$ normal chow diet, $35 \%$ fat and $5 \%$ cholesterol) for 15 weeks. Mice in the $\mathrm{NC}+100 \mathrm{mg} / \mathrm{kg}$ GBE50 group, the HFD $+25 \mathrm{mg} / \mathrm{kg}$ GBE50 group, the HFD+50 $\mathrm{mg} / \mathrm{kg}$ GBE50 group, and the HFD $+100 \mathrm{mg} / \mathrm{kg}$ GBE50 group were given gavage of $100 \mathrm{mg} / \mathrm{kg}, 25 \mathrm{mg} / \mathrm{kg}, 50 \mathrm{mg} / \mathrm{kg}$, and $100 \mathrm{mg} / \mathrm{kg}$ GBE50 every day from 3 to 15 weeks, respectively; the NC group and the NAFLD model group were given gavage of normal saline of the same volume at the same feeding period.

\section{Blood Biochemical Detection}

After the end of the drug regimen, the mice were deprived of water and food for $12 \mathrm{~h}$, and the mice were anesthetized with sodium pentobarbital $(60 \mathrm{mg} / \mathrm{kg})$ by intraperitoneal injection (i.p.). Then, blood was collected from the orbit by using vacuum anticoagulant tubes. The blood samples were placed in vacuum anticoagulant tubes for $10 \mathrm{~min}$ and centrifuged at $1500 \mathrm{~g} / \mathrm{min}$ at room temperature for $20 \mathrm{~min}$ to collect serum. The levels of serum alanine aminotransferase (ALT), aspartate aminotransferase (AST), triglyceride (TG), total cholesterol (TC), free fatty acids (FFA), high-density lipoprotein cholesterol (HDL-C) and lowdensity lipoprotein cholesterol (LDL-C) were measured by biochemical assay according to the corresponding kit's instructions (cat No. C009-3-1, C010-3-1, A110-1-1, A111-1-1, A042-2-1, A112-1-1, A113-1-1; Nanjing Jiancheng Bioengineering Institute, Nanjing, China) by using an automatic biochemical analyzer (AU480, Beckman Coulter, Miami, FL, USA), respectively. The levels of fasting blood glucose (FBG) and fasting insulin (FINS) were quantified by kits assay according to the corresponding kit's instructions (cat No. YC0713, YS02720B; Shanghai YaJi Biotechnology Co., Ltd., 
Shanghai, China). The degree of IR was evaluated by using a homeostasis model assessment of IR index (HOMA-IR). The HOMA-IR was calculated as FBG $(\mathrm{mmol} / \mathrm{L}) \times \mathrm{FINS}(\mu \mathrm{U} / \mathrm{mL}) / 22.5$.

\section{Glucose Tolerance Test (GTT) and Insulin Tolerance Test (ITT) Assays}

For GTT and ITT assays, five mice (12 h overnight-fasted mice) of each group at the end of the drug regimen were intraperitoneally injected with glucose $(1.5 \mathrm{~g} / \mathrm{kg}$, SigmaAldrich, St. Louis, MO, USA) or insulin (1.0 IU/kg, Sigma-Aldrich), then, the blood glucose levels of caudal vein were detected by using a blood glucose monitor (AlphaTRAK, Abbott Molecular, Abbott Park, IL, USA) at 0 (baseline), 30, 60, 90 and $120 \mathrm{~min}$ after the injection.

\section{Liver Tissue Collection}

At the end of the experimental period, all the mice were anesthetized with sodium pentobarbital by i.p. Then, the mice were sacrificed and the livers were harvested. After weighing each liver, it was divided into two parts. One part of liver tissue (left lobe) was used for subsequent biochemical assays and Western blot analysis, while the other part (right lobe) was fixed with $4 \%$ paraformaldehyde for $24 \mathrm{~h}$ and then used for histological measurement and immunohistochemistry.

\section{Liver Tissue Biochemical Detection}

The mouse IL-6, IL- $1 \beta$ and TNF- $\alpha$ ELISA kits (cat No. H007, H002, H052-1; Nanjing Jiancheng Bioengineering Institute) were used to detect the levels of IL-6, IL-1 $\beta$ and TNF- $\alpha$ in liver tissues. After liver tissues were homogenized by RIPA reagent (cat No. P0013C; Beyotime, Shanghai, China), the liver homogenates were collected for ELISA assay according to the corresponding kit's instructions, respectively. Briefly, liver homogenates were added into the plates that had been coated with the corresponding antibodies, and incubated at room temperature for $2 \mathrm{~h}$. Then, the solution was removed and incubated with Biotin-labeled goat anti-rabbit $\operatorname{IgG}(\mathrm{H}+\mathrm{L})$ antibody at $37^{\circ} \mathrm{C}$ for $45 \mathrm{~min}$. Followed, rinsed the well with PBS for 3 times, and incubated with HRP-conjugated Streptavidin antibody at $37^{\circ} \mathrm{C}$ for $30 \mathrm{~min}$. Finally, TMB solution was added and incubated at room temperature for $20 \mathrm{~min}$. The OD values of the wells were measured at wavelength of $490 \mathrm{~nm}$ by using a microplate reader (Bio-Tek, Winooski, VT, USA). Protein concentrations of liver homogenates were quantified by BCA assay (cat No. P0012; Beyotime). According to the protein concentrations of liver homogenates, the contents of hepatic IL-6, IL-1 $\beta$ and TNF- $\alpha$ in unit $\mathrm{mg}$ protein were calculated.

For detection of TC and TG in liver tissues, the liver tissues were homogenized in a 10 -fold volume (v/w) of anhydrous ethanol for $5 \mathrm{~min}$, and the liver homogenates were collected for TC and TG detection by using the corresponding kit (cat No. A110-1-1, A111-1-1; Nanjing Jiancheng Bioengineering Institute) according to the manufacturer's instructions, respectively. For detection of FFA in liver tissues, the liver tissues were homogenized in a 10-fold volume (v/w) of cold PBS for $5 \mathrm{~min}$, and the liver homogenates were collected for FFA detection using an FFA assay kit (cat No. A042-2-1; Nanjing Jiancheng Bioengineering Institute) according to the manufacturer's instruction. The protein concentrations of liver homogenates were measured by BCA assay (Beyotime). According to the protein concentrations of liver homogenates, the contents of hepatic TC, TG and FFA in unit mg protein were calculated.

\section{Hematoxylin-Eosin (HE), Masson Trichrome and Sirius Red Staining}

Paraffin-embedded liver tissues were sliced into $5 \mu \mathrm{m}$ thickness sections. After deparaffinization and hydration, liver sections were stained with HE, Masson Trichrome, and Picrosirius Red according to the corresponding staining kits' instructions (cat No. G1120, G1340, S8060; Solarbio), respectively. The histological features of liver were observed and the staining images were captured by a BX53MRF-S microscope (Olympus). Six random fields of each section were selected, and hepatic steatosis, inflammation and fibrosis of each field were quantified by using the Image J software (version 1.8.0, NIH, USA).

\section{Oil Red O Staining}

Paraffin-embedded liver tissue were sliced into $10 \mu \mathrm{m}$ thickness sections. After deparaffinization and hydration, liver sections were rinsed with $60 \%$ isopropyl alcohol for 20 30 s, followed by staining with Oil red O solution (cat No. G1261; Solarbio) at room temperature for $10 \mathrm{~min}$. The excess dye liquor was slightly washed with 60\% isopropyl alcohol, and then washed with distilled water to remove the isopropyl alcohol. The nucleus was stained with hematoxylin and differentiated with $1 \%$ hydrochloric acid alcohol. Finally, rinsed with water for $10 \mathrm{~min}$ and dried at room temperature. The lipid accumulation in liver tissues 
was observed and the Oil red $\mathrm{O}$ staining images were captured by a BX53MRF-S microscope (Olympus). Six random fields of each section were selected, and the Oil red O-positive staining (lipid droplet) area of each field was counted by using the Image J software (version 1.8.0).

\section{Immunohistochemical Staining}

The procedure of immunohistochemical staining was described as previously. ${ }^{14}$ Briefly, paraffin-embedded liver sections (5 $\mu \mathrm{m}$ thickness) were deparaffinized and rehydrated, then disposed with $3 \% \mathrm{H}_{2} \mathrm{O}_{2}$ for 10 min followed by blocking with $5 \%$ donkey serum for $30 \mathrm{~min}$ at room temperature, finally, incubated with primary antibodies against F4/80 (macrophage marker; cat No. ab16911; 1:200, Abcam, Cambridge, MA, USA), CD45 (leukocyte marker; cat No. \#70257; 1:250, Cell Signaling Technology, Danvers, MA, USA), and Ly6G (neutrophils marker; cat No. \#87048; 1:150, Cell Signaling Technology) overnight at $4{ }^{\circ}$ C. After incubation with the Biotin-labeled goat anti-rabbit IgG $(\mathrm{H}+\mathrm{L})$ and $\mathrm{HRP}$-conjugated Streptavidin antibodies (cat No. PK-4001; ZSGB-BIO Inc, Beijing, China), the immunostaining was visualized by using DAB solution (cat No. SW1040; Solarbio), and the sections were counterstained with hematoxylin solution (cat No. H8070; Solarbio). The inflammatory cell infiltration in liver tissues was observed and the immunostaining images were captured by a BX53MRF-S microscope (Olympus). Six random fields of each section were selected, and the immunostainingpositive area of each field was quantified by using the ImageJ software (version 1.8.0).

\section{Western Blot Analysis}

RIPA reagent (cat No. P0013C; Beyotime) was employed to isolate the total proteins from the liver tissues of each group. Nuclear and cytoplasmic proteins were isolated by using a Nuclear and Cytoplasmic Protein Extraction Kit (cat No. P0027; Beyotime). The concentrations of the isolated proteins were measured by using a BCA Protein Quantitative Kit (cat No. P0012; Beyotime) according to the kit's instruction. Then, the isolated proteins were used to analyzed by Western blot. The protocol of Western blot was described as previously. ${ }^{15}$ The primary antibodies including eukaryotic translation initiation factor- $2 \alpha$ (eIF2 $\alpha$ ) (cat No. sc-133132), activating transcription factor 4 (ATF-4) (cat No. sc-390063), fatty acid synthase (FAS) (cat No. sc-8009), sterol regulatory element-binding protein-1c (SREBP-1c) (cat No. sc-365513), acetylCoenzyme A carboxylase- $\alpha$ (ACC $\alpha$ ) (cat No. sc-271965),
C/EBP homologous protein (CHOP) (cat No. sc-7351) and glucose regulator protein 78 (GRP78) (cat No. sc-13539) (1:1000; all from Santa Cruz, CA, USA), p-eIF2 $\alpha$ (cat No. \#9721), insulin receptor substrates 1 (IRS-1) (cat No. \#2382), p-IRS-1 ${ }^{\text {Tyr608/612 }}$ (cat No. \#3203), p-IRS-1 ${ }^{\text {Ser307 }}$ (cat No. \#2381), p-Akt ${ }^{\mathrm{Thr} 308}$ (cat No. \#13038), p-Akt ${ }^{\mathrm{Ser} 473}$ (cat No. \#4060), AKT (cat No. \#9272), nuclear factor kappa-B (NF-кB) p65 (cat No. \#59674), inhibitor of NF$\kappa \mathrm{B}(\mathrm{I} \kappa \mathrm{B} \alpha$ ) (cat No. \#4812), and p-IкB $\alpha$ (cat No. \#2859) (1:2000; all from Cell Signaling Technology), were used in this experiment. $\beta$-Actin (cat No. AF0003; 1:8000; Beyotime) was used as an internal reference, and the relative expression levels of the target proteins were quantified by using the ImageJ software (version 1.8.0).

\section{Statistical Analysis}

The data were presented as mean \pm standard deviation (SD). Statistical analysis was performed using the SPSS 20.0 software. Comparisons among multiple groups were performed by one-way ANOVA with post hoc Fisher's Least Significant Difference test. $P$ value less than 0.05 was considered to be statistically significant.

\section{Results}

\section{GBE50 Attenuated IR in HFD-Fed Mice}

Glucose metabolism disorder and IR are the typical symptoms of NAFLD. ${ }^{16}$ In this study, FBG and FINS were significantly increased in the NAFLD model group $(P<0.01)$, but were significantly decreased after treatment with GBE50 $(P<0.05$ or 0.01$)$ (Figure $1 \mathrm{~A}$ and B). Furthermore, systemic IR was evaluated by the HOMAIR index, our data (Figure 1C) showed that HFD induced a higher HOMA-IR value $(P<0.01)$, suggesting a significant increased level of IR, which was significantly reduced by GBE50 treatment ( $P<0.05$ or 0.01$)$. The data of GTT and ITT (Figure 1D and E) revealed that there were no differences between $\mathrm{NC}$ group and $\mathrm{NC}+100 \mathrm{mg} / \mathrm{kg}$ GBE50 group; however, GBE50 administration cleared blood glucose efficiently after glucose or insulin injection. Taken together, these data suggested that GBE50 administration could increase glucose clearance and insulin sensitivity.

\section{GBE50 Ameliorated HFD-Induced Hepatic Lipid Metabolism Disorder}

Hepatic lipid metabolism disorder is a key pathological change of NAFLD. ${ }^{10,11,17}$ In this study, HFD not only 


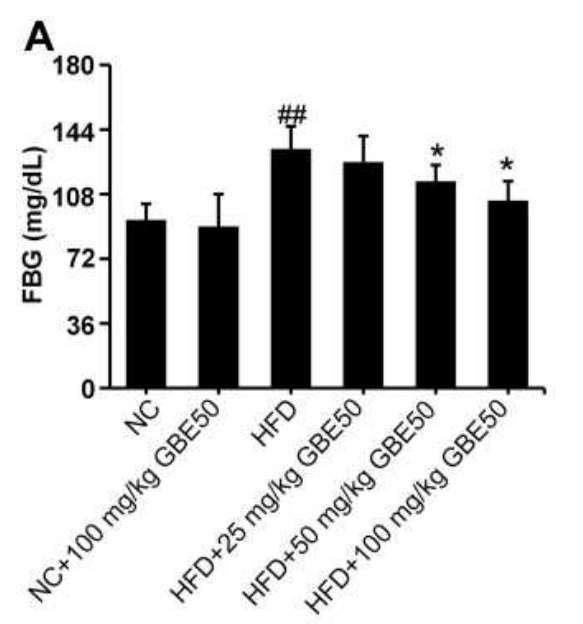

D

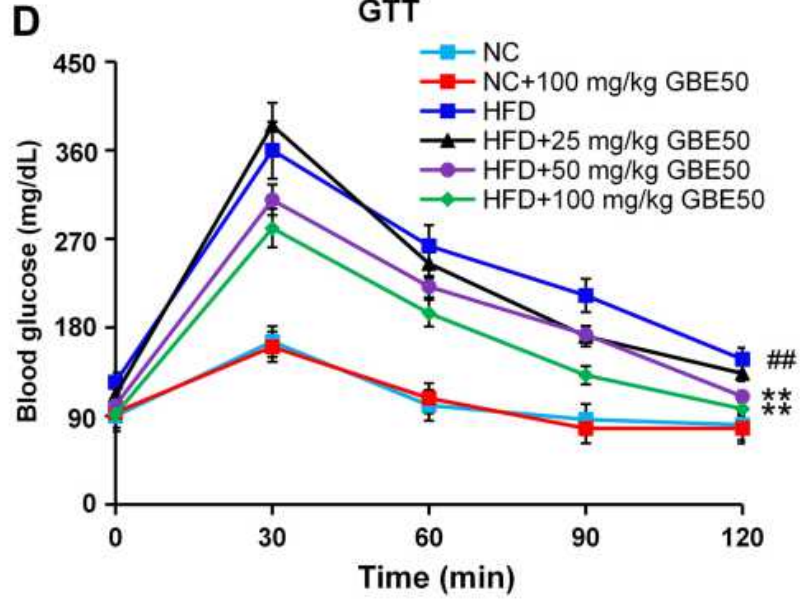

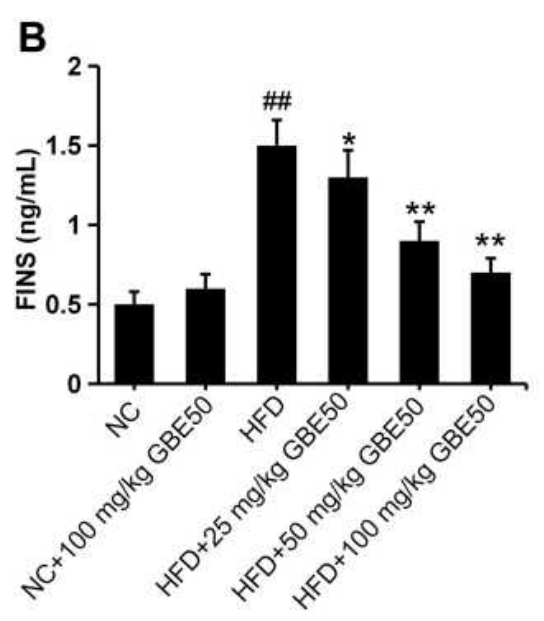

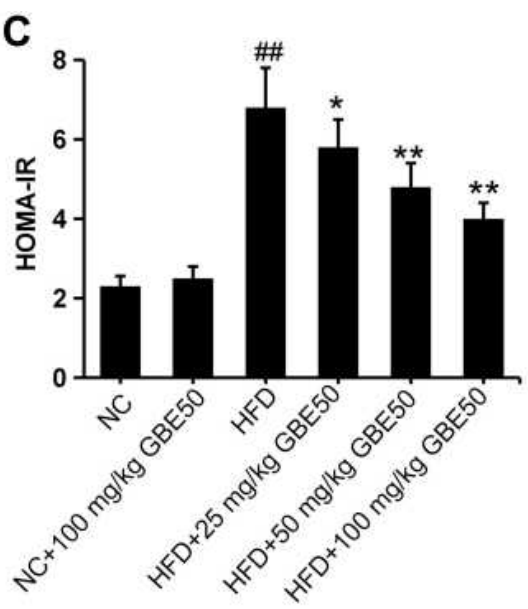

E

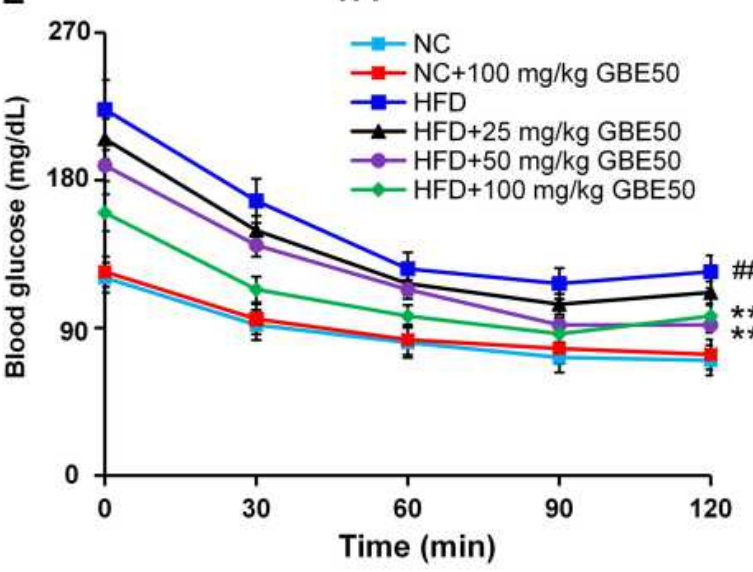

Figure I GBE50 attenuated IR in HFD-fed mice. (A-C) The levels of fasting blood glucose (FBG) (A), fasting insulin (FINS) (B) and the homeostasis model assessment of IR (HOMA-IR) values (C); $n=I I$ mice/group. ( $\mathbf{D}$ and $\mathbf{E}$ ) The results of glucose tolerance test (GTT) (D) and insulin tolerance test (ITT) (E); $n=5$ mice/group. All data are presented as mean $\pm \mathrm{SD}, * P<0.05, * * P<0.0 \mathrm{I}$ vs $\mathrm{HFD}$ alone group; ${ }^{\#} P<0.01$ vs $\mathrm{NC}$ group.

Abbreviations: NC, normal control group; HFD, high-fat diet.

increased hepatosomatic index (liver weight/body weight) (Figure 2A) but also promoted the deposition of lipid droplets (Figure 2B and $\mathrm{C}$ ), which was significantly ameliorated by GBE50 treatment $(P<0.05$ or $0.01)$. Growing research findings showed that the abnormal elevation of TC and TG contributes to the development of NAFLD. ${ }^{18,19}$ Our results showed that serum TG and TC levels in the NAFLD model group were significantly increased $(P<0.01)$, while GBE50 treatment significantly inhibited the HFD-induced elevated TG and TC levels $(P<0.05$ or 0.01$)$ (Figure $2 \mathrm{D}$ and $\mathrm{E})$. This phenomenon was also present in liver tissues (Figure $2 \mathrm{~F}$ and $\mathrm{G})$. LDL-C and HDL-C are also known to be key indicators affecting the progression of NAFLD, so we examined the effect of GBE50 on LDL-C and HDL$\mathrm{C}$ levels in mice fed a HFD diet. As shown in Figure 2H and I, compared with $\mathrm{NC}$ group, serum HDL-C in NAFLD model group was significantly decreased and LDL-C was significantly increased $(P<0.01)$; compared with NAFLD model group, serum HDL-C was significantly increased and LDL-C was significantly decreased after GBE50 treatment $(P<0.05$ or 0.01$)$. In addition, GBE50 markedly inhibited HFD-induced the increase level of FFA in serum and liver tissues (Figure 2J and $\mathrm{K})$. We also examined the fat synthesis-related proteins FAS, SREBP-1c and ACC $\alpha$ in this study, as shown in Supplemental Figure 1, GBE50 could markedly inhibited HFD-induced the increased expression levels of FAS, SREBP-1c and ACC $\alpha(P<0.05$ or 0.01$)$. Overall, these data confirmed that GBE50 administration could ameliorate HFD-induced hepatic lipid metabolism disorder. 


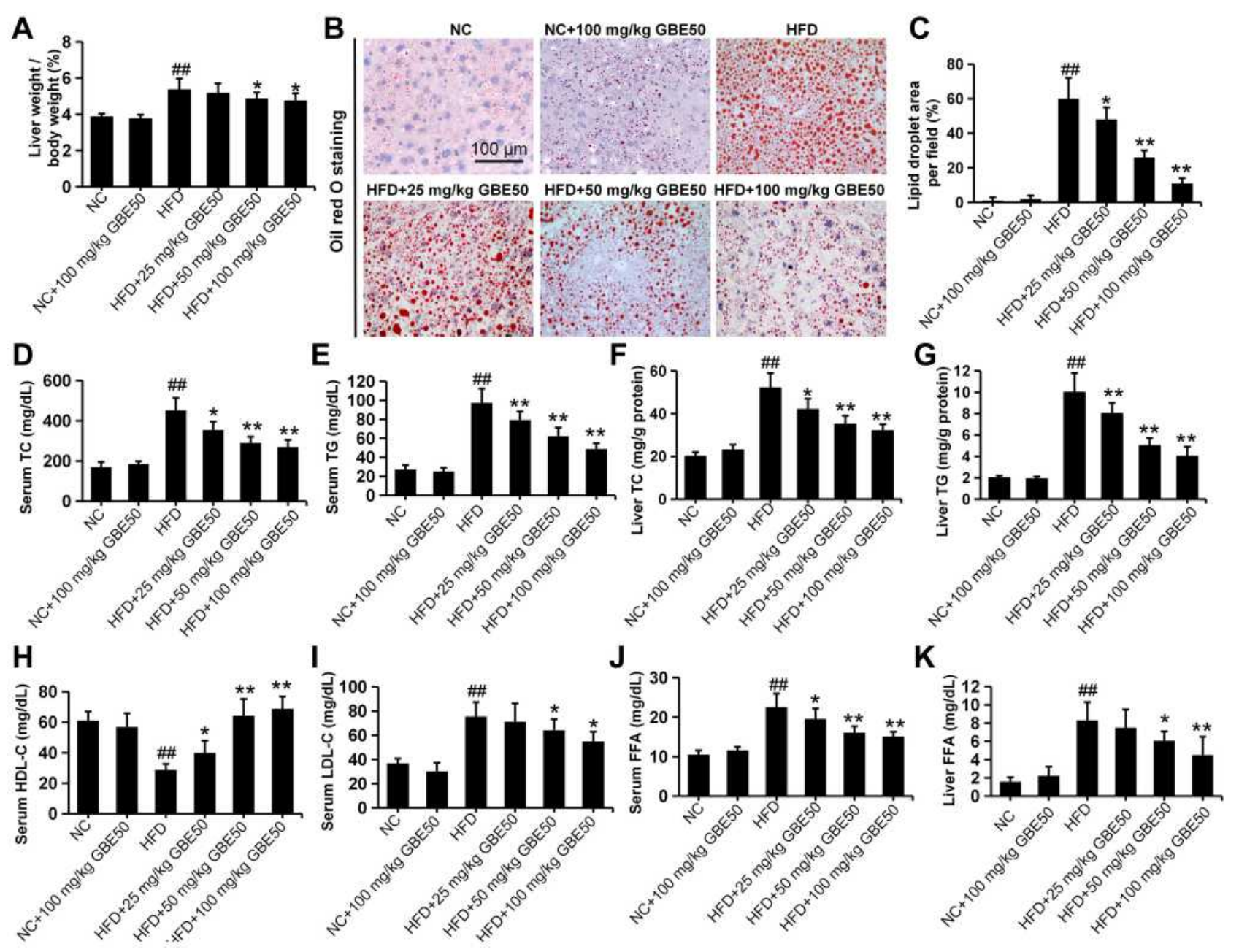

Figure 2 GBE50 ameliorated HFD-induced hepatic lipid metabolism disorder. (A) The results of the ratio of liver weight to body weight. (B and C) Representative Oil red O staining images (B) and the quantification of lipid droplet (C). (D-K) The levels of serum total cholesterol (TC) (D), serum triglyceride (TG) (E), liver TC (F), liver TG (G), serum high-density lipoprotein cholesterol (HDL-C) (H), serum low-density lipoprotein cholesterol (LDL-C) (I), serum free fatty acids (FFA) (J), and liver FFA (K). All data are presented as mean $\pm S D, n=1$ I mice/group, ${ }^{*} P<0.05$, ${ }^{*} * P<0.0$ I vs $\mathrm{HFD}$ alone group; ${ }^{\#} P<0.0 \mathrm{I}$ vs $\mathrm{NC}$ group.

Abbreviations: NC, normal control group; HFD, high-fat diet.

\section{GBE50 Ameliorated HFD-Induced Liver Injury}

We directly observed the liver histopathological changes by HE, Masson Trichrome and Picrosirius Red staining, and the results (Figure 3A-D) showed that, liver tissues in the NAFLD model group presented a large number of vacuolated hepatocyte changes (HE staining) and fibrosis (Masson Trichrome and Picrosirius Red staining), which was significantly attenuated by GBE50 treatment $(P<0.05$ or 0.01$)$. We also evaluated the NAS scores and the degree of fibrosis in various groups. The data (Supplemental Table 1) showed that, compared with the NAFLD model group, the NAS scores and the degree of fibrosis were lower in the GBE50 treatment group, especially in the high-dose group, $72.7 \%$ of the mice without fibrosis $(8 / 11)$. ALT and AST are the two important indicators of liver injury. ${ }^{20}$ To investigate the effect of GBE50 on hepatic injury during NAFLD, we then examined the two indicators in serum. As shown in Figure 3E and F, GBE50 could significantly inhibit the increased activity of ALT and AST induced by HFD $(P<0.05$ or 0.01$)$, suggesting that GBE50 could ameliorate HFD-induced liver injury.

\section{GBE50 Attenuated HFD-Induced Inflammation in Liver}

Chronic inflammation is generally associated with NAFLD and can further promote liver injury. ${ }^{21}$ Therefore, we further examined the inflammatory response in liver tissues in HFD-fed mice. As shown in Figure 4A-D, GBE50 could alleviate HFD-induced infiltration of macrophages and neutrophils $(P<0.05$ or 0.01$)$. This effect of GBE50 

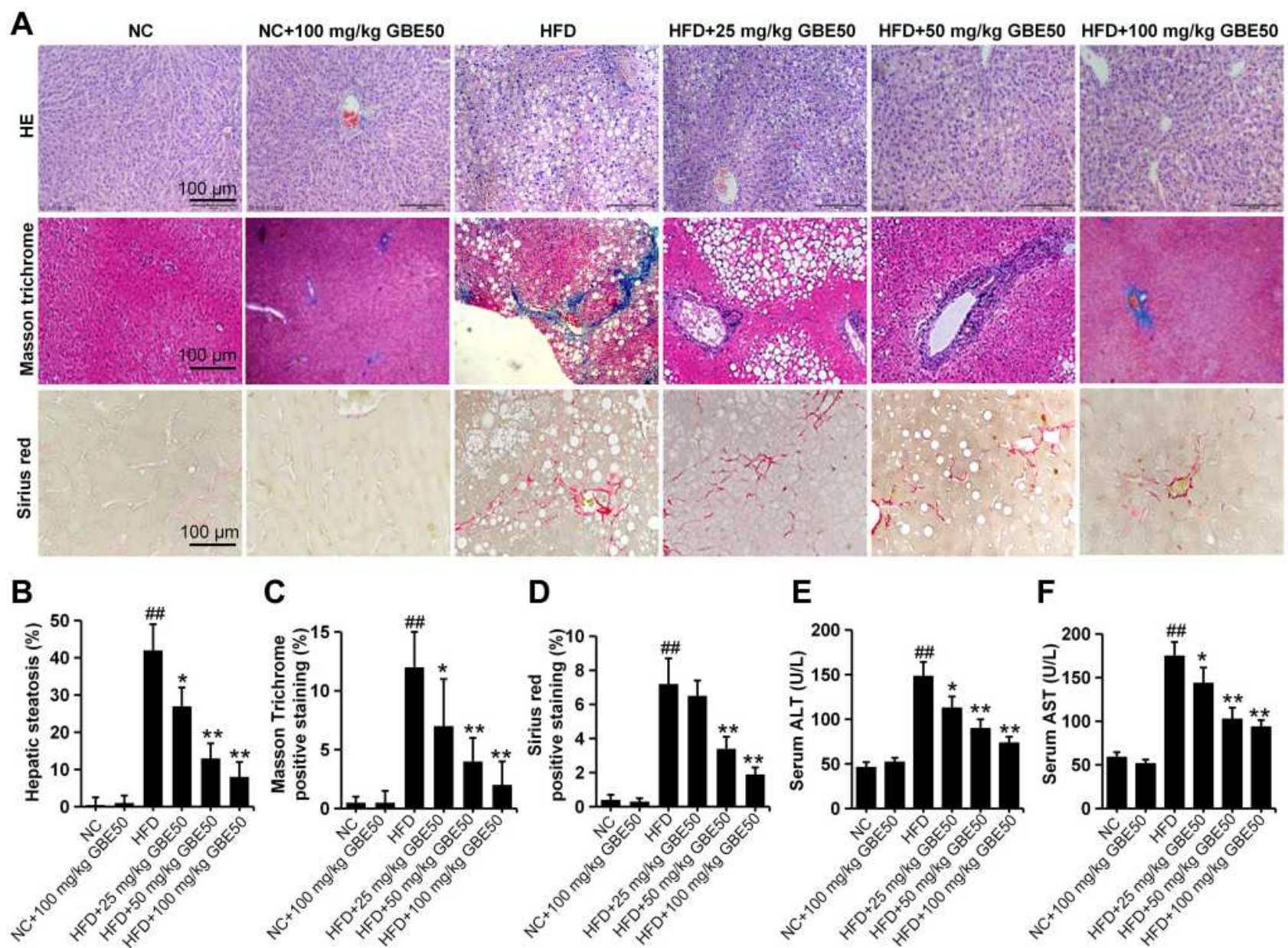

D E

$\mathbf{F}$

Figure 3 GBE50 ameliorated HFD-induced liver injury. (A-D) Representative liver sections images of hematoxylin-eosin (HE) staining, Masson trichrome staining and Sirius red staining (A), the quantitative data of hepatic steatosis from the $\mathrm{HE}$ staining (B), Masson trichrome positive staining area (C), and Sirius red staining positive staining area (D). (E and F) The levels of serum alanine aminotransferase (ALT) (E) and aspartate aminotransferase (AST) (F). All data are presented as mean \pm SD, $n=1 /$ mice/group, $* P<0.05, * * P<0.01$ vs HFD alone group; ${ }^{\#} P<0.01$ vs $N C$ group.

Abbreviations: NC, normal control group; HFD, high-fat diet.

was further confirmed by ELISA, and the expression levels of pro-inflammatory cytokines (IL-6, IL-1 $\beta$ and TNF- $\alpha$ ) in liver tissues of HFD-fed mice were significantly decreased after GBE50 treatment $(P<0.01)$ (Figure 4E-G). As we know that NF- $\mathrm{kB}$ signaling pathway is one of the most important signaling pathways in the regulation of inflammatory response. Our data (Supplemental Figure 2) show that GBE50 could significantly promote the nuclear translocation of NF- $\kappa \mathrm{B}$ p 65 and decreased the phosphorylation level of I $\kappa \mathrm{B} \alpha$ in liver tissues of HFD-fed mice $(P<0.05$ or 0.01$)$.

\section{GBE50 Regulated the Hepatic Insulin Signaling and ERS in HFD-Fed Mice}

IR is also a key pathological mechanism leading to NAFLD. ${ }^{10,11,16}$ To assess the hepatoprotective of GBE50 on NAFLD, the activation of IRS-1 pathway was determined. As seen in Figure 5A-D, GBE50 administration activated insulin signaling in HFD-fed mice, as evidenced by mild increase in the expression levels of $\mathrm{p}-\mathrm{IRS}-1^{\text {Tyr608 }}$ and reduced the expression levels of p-IRS-1 ${ }^{\mathrm{Ser} 307}$ in the liver tissues; moreover, an acute injection of insulin dramatically activated insulin signaling, which was further enhanced after GBE50 administration. Akt signaling pathway is one of the most important downstream signals of IRS- $1^{22,23}$; therefore, we further tested the activity of Akt. As shown in Figure 5E-G, GBE50 could significantly decrease the phosphorylation level of Akt at Thr308/ Ser473 in liver tissues of HFD-fed mice $(P<0.05$ or 0.01$)$.

ERS response is one of the key steps leading to liver injury in NAFLD. ${ }^{10,11,17}$ We then examined the ERSrelated proteins p-eIF2 $\alpha$, ATF-4, CHOP and GRP78. The 


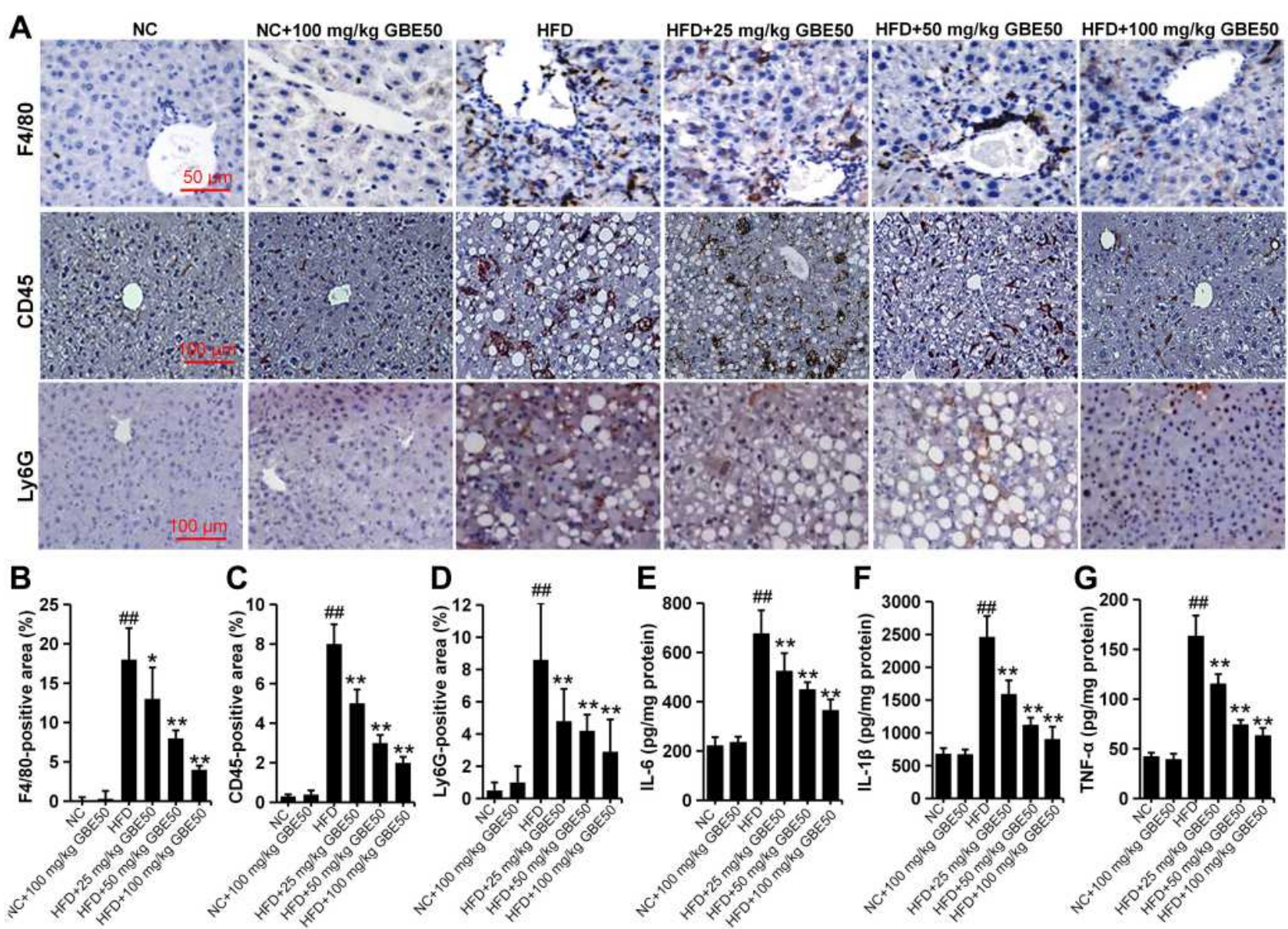

Figure 4 GBE50 attenuated HFD-induced inflammatory cell infiltration and the expression levels of pro-inflammatory cytokines in liver. (A-D) Representative immunohistochemical staining images of the expressions of F4/80, CD45, and Ly6G (A), and the quantification of F4/80 (B), CD45 (C), and Ly6G (D) positive staining areas in liver tissues. (E-G) The levels of IL-6 (E), IL-I $\beta(\mathbf{F})$ and TNF- $\alpha(\mathbf{G})$ in liver tissues. All data are presented as mean \pm SD, $n=1$ I mice/group, $* P<0.05$, **P< $<0.01$ vs HFD alone group; ${ }^{\#} \mathrm{P}<0.01$ vs NC group.

Abbreviations: NC, normal control group; HFD, high-fat diet.

results showed HFD induced higher expression levels of p-eIF2 $\alpha$, ATF-4, CHOP and GRP78, suggesting a significant increase level of ERS, which was significantly reduced by GBE50 treatment $(P<0.01)$ (Figure $6 \mathrm{~A}-\mathrm{G})$.

\section{Discussion}

NAFLD has become a serious public health problem, which affects about $25 \%$ of the adult population worldwide. $^{7}$ Unfortunately, there is no specific drug currently available for the treatment of NAFLD. Natural Chinese herbal medicines have been proven to have a wide range of pharmacological effects that are safe, effective and low toxicity. ${ }^{24}$ Screening small-molecule compounds or monomers with anti-inflammatory, antioxidation, maintenance of lipid homeostasis and enhancement of insulin sensitivity from natural Chinese medicinal materials may be a potential strategy to search for antiNAFLD drugs. Our present study demonstrated that, GBE50, the new Ginkgo biloba extracts, had the pharmacological effects of inhibiting IR, ameliorating hepatic steatosis and relieving liver injury in HFD-fed mice. AAndThese data here suggested that GBE50 was a potential anti-NAFLD drug.

Hepatic steatosis and lipid accumulation are the key pathological changes in NAFLD, and also the key factors that trigger subsequent liver function decline and liver injury. ${ }^{10,11,17}$ Metabolic disorders induced by HFD can increase the abnormal levels of TC, TG and LDL-C, and decrease the level of HDL-C. Especially, the abnormal deposition of TC, TG and LDL-C in the liver can promote hepatic steatosis, and the continuous accumulation of TC, TG and LDL-C and the continuous decrease of HDL-C also can promote the sensitivity of the liver to 


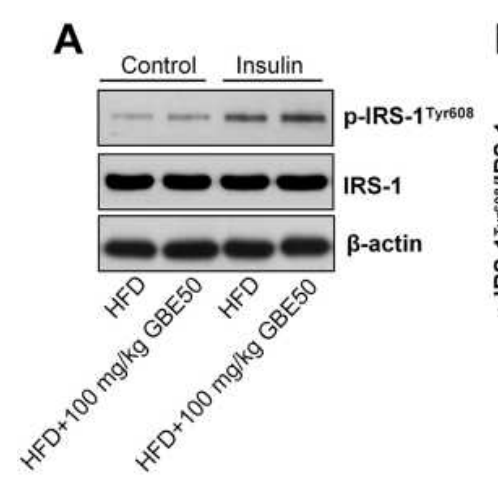

E

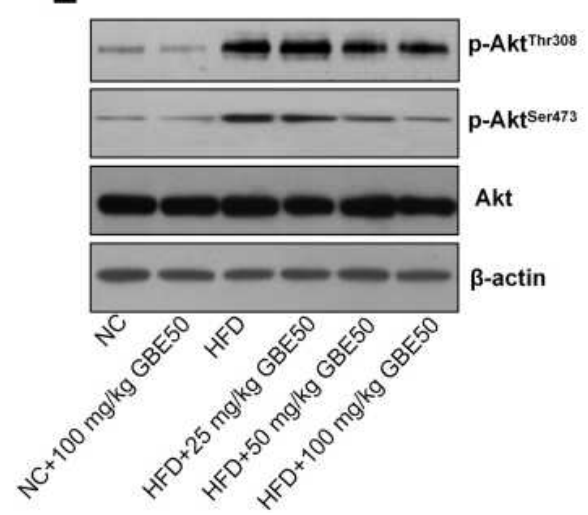

B

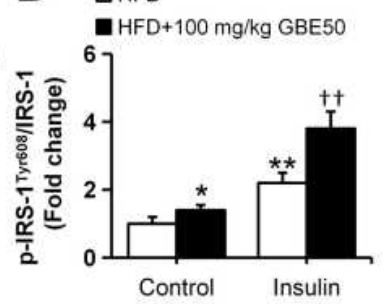

C

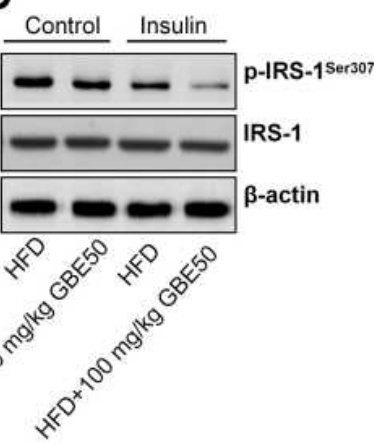

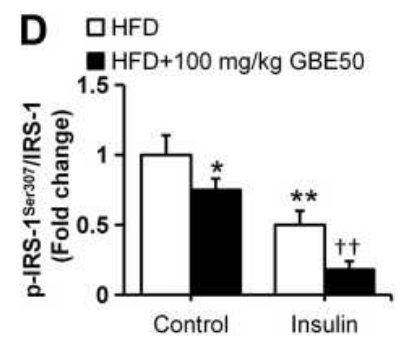

G
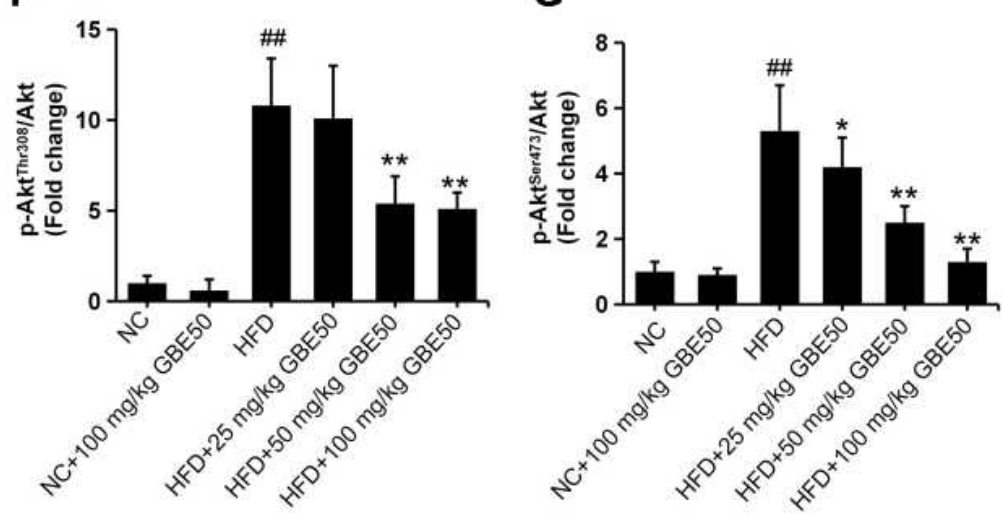

Figure 5 GBE50 promoted IRS-I signal activation and inhibited Akt signal activation in HFD-fed mice. (A-D) The phosphorylation levels of IRS-I (Tyr608) (A and B) and IRS-I (Ser307) (C and D) under baseline (control) and in response to an intraperitoneal injection of insulin (I.0 IU/kg for I5 min) in HFD alone group and HFD+ I00 mg/kg GBE50 group; $n=5$ mice/group. (E-G) The expression levels of p-Akt (Thr308) (E and $\mathbf{F}$ ) and p-Akt (Ser473) (E and $\mathbf{G})$ proteins in liver tissues in various of groups were detected by Western blot; $n=6$ mice/group. All data are presented as mean $\pm S D, * P<0.05, * * P<0.0$ I vs HFD alone group; ${ }^{\dagger \dagger} P<0.0$ I vs $H F D+i n s u l i n$ group; ${ }^{\#} P<0.0$ I vs $N C$ group.

Abbreviations: NC, normal control group; HFD, high-fat diet.

inflammation and the progression of NAFLD. ${ }^{18,19}$ This study showed that GBE50 reduced TC, TG and LDL-C levels, increased HDL-C levels, and also alleviated hepatic steatosis and lipid accumulation in HFD-fed mice. HFD can lead to an increased level of circulation FFA, while the excessively high levels of circulation FFA will increase the synthesis of TG and TC in liver, and cause IR, which will lead to the disorder of lipid metabolism, and finally lead to liver lipid deposition. ${ }^{25,26}$ In addition, abnormal amounts of FFA accumulate in liver cells, which can cause lipid peroxidation and oxidative stress, resulting in degeneration and necrosis of liver cells and inflammatory response. ${ }^{25,26}$ In the current study, we found that GBE50 decreased the FFA level in serum and liver tissues in HFD-fed mice. To investigate the mechanism of GBE50 inhibits lipid accumulation, the expression levels of FAS, SREBP-1c and ACC $\alpha$ proteins were evaluated in this study. Our results found that the expression levels of FAS, SREBP-1c and $\mathrm{ACC} \alpha$ proteins were obviously decreased in HFD-fed mice after GBE50 treatment, suggesting that GBE50 decreased de novo lipid synthesis may reduce the expression levels of FAS, SREBP-1c and ACC $\alpha$.

Liver injury and liver dysfunction are the important driving factors for the progression of liver diseases, including NAFLD. ${ }^{10,11,17,27,28}$ ALT and AST are important indicators for the evaluation of liver injury and liver function in the biochemical examination of NAFLD, and long-term HFD can cause liver injury and liver function decline and increase the level of ALT and AST. ${ }^{20}$ The results of this study showed that GBE50 could significantly reduce ALT and AST levels in serum of HFD-fed mice, suggesting that GBE50 could reduce liver injury induced by HFD. In addition, liver steatosis induced by HFD is closely related to chronic liver inflammation, which increases the sensitivity of liver cells to proinflammatory cytokines mediated injury, and persistent chronic inflammation further exacerbates the progression of NAFLD. ${ }^{10,11,16,17,21}$ Therefore, this study further observed whether GBE50 had a protective effect on the inflammatory response of liver in NAFLD, and the 

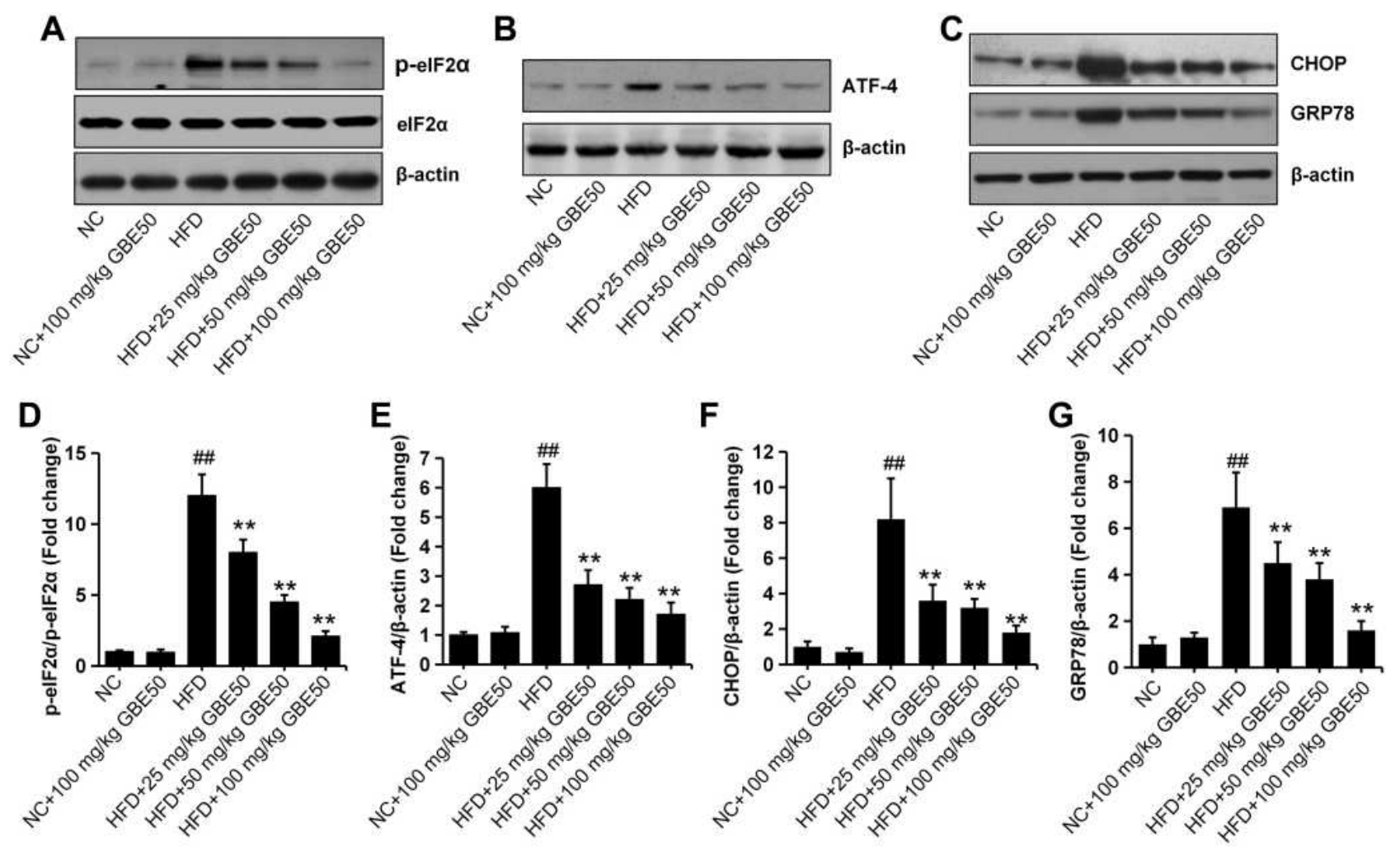

Figure 6 GBE50 attenuated endoplasmic reticulum stress in HFD-fed mice. (A-C) Representative Western blot bands of the expressions of P-elF2 $\alpha$, elF2 $\alpha$, ATF-4, CHOP and GRP78 in liver tissues in various of groups. (D-G) The quantification of the expression levels of p-elF2 (D), ATF-4 (E), CHOP (F) and GRP78 (G). All data are presented as mean $\pm S D, n=6$ mice/group, ${ }^{* * P}<0.01$ vs HFD alone group; ${ }^{*} P<0.01$ vs $N C$ group.

Abbreviations: NC, normal control group; HFD, high-fat diet.

results showed that GBE50 alleviated the pathological changes of inflammatory cell infiltration and reduced the levels of pro-inflammatory cytokines IL-6, IL-1 $\beta$ and TNF- $\alpha$ in liver tissues in HFD-fed mice, indicating that GBE50 could effectively inhibit liver inflammation caused by HFD. To explore the mechanism by which GBE50 inhibits HFD-induced liver injury, we analyzed the activity of NF- $\mathrm{KB}$, a classical signal that affected the inflammatory response. HFD has been reported to activate NF- $\kappa \mathrm{B}$ signal and further aggravated the progression of NAFLD through NF- $\mathrm{KB}$ signal mediated inflammatory response and fibrosis. ${ }^{29,30}$ Inhibition of $\mathrm{NF}-\mathrm{KB}$ signaling activity can reduce the production of pro-inflammatory factors, liver injury and block the progression of NAFLD. ${ }^{31}$ In this study, we found that GBE50 attenuated NF- $\mathrm{KB}$ signaling activity in liver tissues of HFD-fed mice, suggesting that GBE50 may control liver inflammatory response and liver injury of NAFLD through negative regulation of NF- $\mathrm{KB}$ signaling activity.

IR, as one of the initiating factors of NAFLD, is defined as a reduced sensitivity to insulin and associated with defective insulin signal transduction. ${ }^{10,11,16}$ Insulin plays an important role in regulating glucose and lipid homeostasis in the liver. ${ }^{16}$ IRS-1 is the main effector of insulin receptors and is responsible for insulin signal transduction. ${ }^{10,11,16}$ When insulin binds to insulin receptor, phosphorylation of IRS-1 at Tyr608 can activate insulin pathway, in contrast, phosphorylation of IRS-1 at Ser307 is considered to block the insulin signaling, leading to IR. ${ }^{16,32}$ In the current study, the data showed that GBE50 enhanced phosphorylation of IRS-1 at Tyr608 and further inhibited phosphorylation at Ser307 induced by acute injection of insulin, suggesting that GBE50 could attenuate IR by promoting insulin signaling activation in HFD-fed mice.

ERS response is one of the major toxic events leading to liver injury in NAFLD. ${ }^{10,11,17}$ Hypoxia, lipid overloading, inflammation and other pathological factors can disrupt ER homeostasis, resulting in the accumulation of unfolded and misfolded proteins, which can lead to ERS in hepatocytes. ${ }^{33,34}$ In response to this stress, unfolded protein response (UPR) is activated. The UPR involves 3 transmembrane ER pressure sensors: inositol-requiring 
enzyme 1 (IRE1), (PKR)-like ER kinase (PERK) and ATF-6. ${ }^{35}$ Among them, PERK plays an important role in the corresponding ERS response. When ERS is activated, PERK is separated from GRP78, and then, the cytoplasmic eIF2 $\alpha$ is phosphorylated. ${ }^{36}$ The phosphorylated eIF2 $\alpha$ can inhibit protein translation or activate ATF-4 to regulate target gene transcriptional level in response to ER folding load. ${ }^{37}$ It has been found that activation of eIF2/ATF-4 signals could promote adipogenic differentiation and intracellular lipid synthesis of breast epithelial cells by upregulating the expression of FAS. ${ }^{38}$ In animal experiment, Oyadomari et $\mathrm{al}^{39}$ observed that ERS agonist-induced mice presented fatty liver, while eIF2 dephosphorylation significantly ameliorated the fatty liver. Furthermore, phosphorylation of eIF $2 \alpha$ could up-regulate the expression of ATF-4, and then activate the expression of SREBP-1c, ACC2, FAS and other lipid-synthesis-related genes. ${ }^{40}$ ATF-4 knockout was resistant to HFD-induced obesity, hypertriglyceridemia, and NAFLD in mice. ${ }^{41}$ In addition, high levels of phosphorylated eIF $2 \alpha$ in the liver can exacerbate IR and enhance NF- $\mathrm{B}$ activity in NAFLD, which can further aggravate hepatic steatosis and liver injury. ${ }^{33,42,43}$ The above evidences indicated the role of eIF2/ATF-4 in hepatic lipid synthesis and metabolism, IR and liver injury in NAFLD. The results of this study also showed that p-eIF2, ATF-4, CHOP and GRP78 proteins were highly expressed in liver tissues of HFD-fed mice, while they were all decreased after GBE50 treatment, suggesting that GBE50 could ameliorate HFD-induced NAFLD by weakening ERS.

In conclusion, HFD-induced NAFLD mice were characterized by lipid accumulation and hepatic steatosis, accompanied by liver injury, inflammation, glucose intolerance and IR, which were significantly ameliorated after GBE50 treatment. The therapeutic effects of GBE50 on NAFLD may be related to the strengthening of IRS-1 signal activation and the weakening of $N F-\kappa B$ and ERS signal activation. These findings suggested that GBE50 may be a potentially powerful therapeutic agent for NAFLD.

\section{Compliance with Ethical Standards}

All animal experiments were approved and performed according to the guidelines of the institutional animal care and use committee of the Hunan Provincial People's Hospital, with the protocol number: HNPH/IAEC/LS/ 2018-102.

\section{Consent for Publication}

This study is approved by all authors for publication.

\section{Author Contributions}

All authors made a significant contribution to the work reported, whether that is in the conception, study design, execution, acquisition of data, analysis and interpretation, or in all these areas; took part in drafting, revising or critically reviewing the article; gave final approval of the version to be published; have agreed on the journal to which the article has been submitted; and agree to be accountable for all aspects of the work.

\section{Funding}

This work was supported by the Natural Science Foundation of Hunan Province (No. 2018JJ6020) and the Foundation of Health and Family Planning Commission of Hunan Province (No. B2019067).

\section{Disclosure}

The authors have declared that no competing interests exist.

\section{References}

1. Sookoian S, Pirola CJ, Chan C. Systems biology elucidates common pathogenic mechanisms between nonalcoholic and alcoholic-fatty liver disease. PLoS One. 2013;8(3):e58895. doi:10.1371/journal. pone. 0058895

2. Neuschwander-Tetri BA. Non-alcoholic fatty liver disease. BMC Med. 2017;15(1):45. doi:10.1186/s12916-017-0806-8

3. Zhao ZY, Liu D, Cao WJ, et al. Association between IgG N-glycans and nonalcoholic fatty liver disease in Han Chinese. Biomed Environ Sci. 2018;31(6):454-458. doi:10.3967/bes2018.059

4. Lonardo A, Nascimbeni F, Maurantonio M, Marrazzo A, Rinaldi L, Adinolfi LE. Nonalcoholic fatty liver disease: evolving paradigms. World J Gastroenterol. 2017;23:6571-6592. doi:10.3748/wjg.v23. i36.6571

5. Younossi ZM. Non-alcoholic fatty liver disease - a global public health perspective. $J$ Hepatol. 2019;70(3):531-544. doi:10.1016/j. jhep.2018.10.033

6. Kim JY, Cho J, Yang HR. Biochemical predictors of early onset non-alcoholic fatty liver disease in young children with obesity. J Korean Med Sci. 2018;33(16):e122. doi:10.3346/jkms.2018.33.e122

7. Perumpail BJ, Khan MA, Yoo ER, Cholankeril G, Kim D, Ahmed A. Clinical epidemiology and disease burden of nonalcoholic fatty liver disease. World J Gastroenterol. 2017;23:8263-8276. doi:10.3748/wjg. v23.i47.8263

8. Hartmann P, Schnabl B. Risk factors for progression of and treatment options for NAFLD in children. Clin Liver Dis (Hoboken). 2018;11 (1):11-15. doi:10.1002/cld.685

9. Cole BK, Feaver RE, Wamhoff BR, Dash A. Non-alcoholic fatty liver disease (NAFLD) models in drug discovery. Expert Opin Drug Discov. 2018;13(2):193-205. doi:10.1080/17460441.2018.1410135 
10. Manne V, Handa P, Kowdley KV. Pathophysiology of nonalcoholic fatty liver disease/nonalcoholic steatohepatitis. Clin Liver Dis. 2018;22(1):23-37. doi:10.1016/j.cld.2017.08.007

11. Bessone F, Razori MV, Roma MG. Molecular pathways of nonalcoholic fatty liver disease development and progression. Cell Mol Life Sci. 2019;76(1):99-128.

12. Lu S, Guo X, Zhao P. Effect of Ginkgo biloba extract 50 on immunity and antioxidant enzyme activities in ischemia reperfusion rats. Molecules. 2011;16(11):9194-9206. doi:10.3390/molecules16119194

13. He GY, Yuan CG, Hao L, Xu Y, Zhang ZX. GBE50 attenuates inflammatory response by inhibiting the $\mathrm{p} 38$ MAPK and NF- $\kappa B$ pathways in LPS-stimulated microglial cells. Evid Based Complement Alternat Med. 2014;2014:368598. doi:10.1155/2014/ 368598

14. Han C, Ren Y, Jia Y, Kong L, Eerdun T, Wu L. The effective mode of growth and differentiation factor- 5 in promoting the chondrogenic differentiation of adipose-derived stromal cells. Cell Tissue Bank. 2016;17(1):105-115. doi:10.1007/s10561-015-9517-6

15. Huang EW, Xue SJ, Zhang Z, Zhou JG, Guan YY, Tang YB. Vinpocetine inhibits breast cancer cells growth in vitro and in vivo. Apoptosis. 2012;17(10):1120-1130. doi:10.1007/s10495-012-0743-0

16. Buzzetti E, Pinzani M, Tsochatzis EA. The multiple-hit pathogenesis of non-alcoholic fatty liver disease (NAFLD). Metabolism. 2016;65 (8):1038-1048. doi:10.1016/j.metabol.2015.12.012

17. Lebeaupin C, Vallée D, Hazari Y, Hetz C, Chevet E, Bailly-Maitre B. Endoplasmic reticulum stress signalling and the pathogenesis of non-alcoholic fatty liver disease. $J$ Hepatol. 2018;69(4):927-947. doi:10.1016/j.jhep.2018.06.008

18. Marra F, Svegliati-Baroni G. Lipotoxicity and the gut-liver axis in NASH pathogenesis. J Hepatol. 2018;68(2):280-295. doi:10.1016/j. jhep.2017.11.014

19. Csak T, Ganz M, Pespisa J, Kodys K, Dolganiuc A, Szabo G. Fatty acid and endotoxin activate inflammasomes in mouse hepatocytes that release danger signals to stimulate immune cells. Hepatology. 2011;54:133-144. doi:10.1002/hep.24341

20. Kwo PY, Cohen SM, Lim JK. ACG clinical guideline: evaluation of abnormal liver chemistries. Am J Gastroenterol. 2017;112(1):18-35. doi:10.1038/ajg.2016.517

21. Alisi A, Bedogni G, De Vito R, Comparcola D, Manco M, Nobili V. Relationship between portal chronic inflammation and disease severity in paediatric non-alcoholic fatty liver disease. Dig Liver Dis. 2011;43(2):143-146. doi:10.1016/j.dld.2010.05.007

22. Fan Y, He Z, Wang W, et al. Tangganjian decoction ameliorates type 2 diabetes mellitus and nonalcoholic fatty liver disease in rats by activating the IRS/PI3K/AKT signaling pathway. Biomed Pharmacother. 2018;106:733-737. doi:10.1016/j.biopha.2018.06.089

23. Guo JW, Liu X, Zhang TT, et al. Hepatocyte TMEM16A deletion retards NAFLD progression by ameliorating hepatic glucose metabolic disorder. Adv Sci (Weinh). 2020;7(10):1903657. doi:10.1002/ advs.201903657

24. Wu WY, Yang WZ, Hou JJ, De DA. Current status and future perspective in the globalization of Traditional Chinese Medicines. World J Tradit Chin Med. 2015;1(1):1-4. doi:10.15806/j.issn.23118571.2014.0027

25. Staehr P, Hother-Nielsen O, Landau BR, Chandramouli V, Holst JJ, Beck-Nielsen $\mathrm{H}$. Effects of free fatty acids perse on glucose production, gluconeogenesis, and glycogenolysis. Diabetes. 2003;52:260-267. doi:10.2337/diabetes.52.2.260

26. Samuel VT, Shulman GI. Mechanisms for insulin resistance: common threads and missing links. Cell. 2012;148:852-871. doi:10.1016/ j.cell.2012.02.017

27. Shirpoor A, Heshmati E, Kheradmand F, et al. Increased hepatic FAT/ CD36, PTP1B and decreased HNF4A expression contributes to dyslipidemia associated with ethanol-induced liver dysfunction: rescue effect of ginger extract. Biomed Pharmacother. 2018;105:144-150. doi:10.1016/j.biopha.2018.05.121
28. Amini M, Saboory E, Pourheydar B, Bagheri M, Naderi R. Involvement of endocannabinoid system, inflammation and apoptosis in diabetes induced liver injury: role of 5-HT3 receptor antagonist. Int Immunopharmacol. 2020;79:106158. doi:10.1016/j. intimp.2019.106158

29. Xu B, Jiang M, Chu Y, et al. Gasdermin D plays a key role as a pyroptosis executor of non-alcoholic steatohepatitis in humans and mice. $J$ Hepatol. 2018;68(4):773-782. doi:10.1016/j. jhep.2017.11.040

30. Feng D, Zou J, Su D, et al. Curcumin prevents high-fat diet-induced hepatic steatosis in ApoE(-/-) mice by improving intestinal barrier function and reducing endotoxin and liver TLR4/NF-kappaB inflammation. Nutr Metab (Lond). 2019;16:79. doi:10.1186/s12986019-0410-3

31. Hao YR, Tang FJ, Zhang X, Wang H. Suppression of NF-kappaB activation by PDLIM2 restrains hepatic lipogenesis and inflammation in high fat diet induced mice. Biochem Biophys Res Commun. 2018;503(2):564-571. doi:10.1016/j.bbrc.2018.05.187

32. Copps KD, White MF. Regulation of insulin sensitivity by serine/ threonine phosphorylation of insulin receptor substrate proteins IRS1 and IRS2. Diabetologia. 2012;55(10):2565-2582. doi:10.1007/ s00125-012-2644-8

33. Sharma RS, Harrison DJ, Kisielewski D, et al. Experimental nonalcoholic steatohepatitis and liver fibrosis are ameliorated by pharmacologic activation of Nrf2 (NF-E2 p45-Related Factor 2). Cell Mol Gastroenterol Hepatol. 2017;5(3):367-398. doi:10.1016/j. jcmgh.2017.11.016

34. Duvigneau JC, Luís A, Gorman AM, et al. Crosstalk between inflammatory mediators and endoplasmic reticulum stress in liver diseases. Cytokine. 2019;124:154577. doi:10.1016/j.cyto.2018.10.018

35. Hassler J, Cao SS, Kaufman RJ. IRE1, a double-edged sword in pre-miRNA slicing and cell death. Dev Cell. 2012;23(5):921-923. doi:10.1016/j.devcel.2012.10.025

36. Lee YS, Lee DH, Choudry HA, Bartlett DL, Lee YJ. Ferroptosisinduced endoplasmic reticulum stress: cross-talk between ferroptosis and apoptosis. Mol Cancer Res. 2018;16(7):1073-1076. doi:10.1158/ 1541-7786.MCR-18-0055

37. Guo H, Li H, Wang B, et al. Protective effects of glucagon-like peptide-1 analog on renal tubular injury in mice on high-fat diet. Cell Physiol Biochem. 2017;41(3):1113-1124. doi:10.1159/ 000464118

38. Hazari YM, Habib M, Bashir S, et al. Natural osmolytes alleviate GRP78 and ATF-4 levels: corroboration for potential modulators of unfolded protein response. Life Sci. 2016;146:148-153. doi:10.1016/ j.1fs.2016.01.002

39. Oyadomari S, Harding HP, Zhang Y, Oyadomari M, Ron D. Dephosphorylation of translation initiation factor 2alpha enhances glucose tolerance and attenuates hepatosteatosis in mice. Cell Metab. 2008;7(6):520-532. doi:10.1016/j.cmet.2008.04.011

40. Enos RT, Velázquez KT, Carson MS, et al. A low dose of dietary quercetin fails to protect against the development of an obese phenotype in mice. PLoS One. 2016;11(12):e0167979. doi:10.1371/journal.pone. 0167979

41. Cho EJ, Yoon JH, Kwak MS, et al. Tauroursodeoxycholic acid attenuates progression of steatohepatitis in mice fed a methionine-choline-deficient diet. Dig Dis Sci. 2014;59 (7):1461-1474. doi:10.1007/s10620-014-3217-0

42. Nishio N, Isobe K. GADD34-deficient mice develop obesity, nonalcoholic fatty liver disease, hepatic carcinoma and insulin resistance. Sci Rep. 2015;5:13519. doi:10.1038/srep13519

43. Zhang X, Han J, Man K, et al. CXC chemokine receptor 3 promotes steatohepatitis in mice through mediating inflammatory cytokines, macrophages and autophagy. $J$ Hepatol. 2016;64(1):160-170. doi:10.1016/j.jhep.2015.09.005 


\section{Publish your work in this journal}

The Journal of Inflammation Research is an international, peerreviewed open-access journal that welcomes laboratory and clinical findings on the molecular basis, cell biology and pharmacology of inflammation including original research, reviews, symposium reports, hypothesis formation and commentaries on: acute/chronic inflammation; mediators of inflammation; cellular processes; molecular mechanisms; pharmacology and novel anti-inflammatory drugs; clinical conditions involving inflammation. The manuscript management system is completely online and includes a very quick and fair peerreview system. Visit http://www.dovepress.com/testimonials.php to read real quotes from published authors.

Submit your manuscript here: https://www.dovepress.com/journal-of-inflammation-research-journal 